\title{
Effects of adherent Lactobacillus cultures on growth, weight of organs and intestinal microflora and volatile fatty acids in broilers
}

\begin{abstract}
A total of 180 1-day old Arbor Acres chicks was used to investigate the effects of a single L. acidophilus I 26 strain or a mixture of 12 Lactobacillus cultures on the production performance, weight of organs, and intestinal microflora and VFA of broilers. The chicks were assigned randomly into three groups with 60 chicks per treatment. The three dietary treatments were: (i) basal diet (acted as control); (ii) basal diet $+1 \mathrm{~g} \mathrm{~kg}-1 \mathrm{~L}$. acidophilus I 26; and (iii) basal diet+1 $\mathrm{g} \mathrm{kg}-1$ mixture of 12 Lactobacillus strains. The results showed that the addition of either a single L. acidophilus I 26 strain or a mixture of 12 Lactobacillus cultures to the basal diet increased significantly $(\mathrm{P}<0.05)$ the body weight and feed:gain ratio of broilers for 0-6 weeks. Supplementing the Lactobacillus cultures, singly or in a mixture, in the diet of broilers also decreased significantly $(\mathrm{P}<0.05)$ the numbers of coliforms in the cecum 10 and 20 days after feeding, increased significantly $(\mathrm{P}<0.05)$ the total VFA in the ileum and cecum, and lowered the cecal $\mathrm{pH}$ values. However, the addition of the Lactobacillus cultures in the diets did not increase significantly the lactobacilli population in the ileum and cecum of broilers, except for 30 days after feeding. There were also no significant differences in the populations of total anaerobes, total aerobes, Bifidobacteria and Streptococcus in the ileal and cecal contents of chickens fed with or without Lactobacillus cultures. No significant differences were found in the weight of the liver, spleen, bursa, gizzard, duodenum, jeju-ileum and total small intestine of broilers given the different dietary treatments.
\end{abstract}

Keyword: Chicken; Lactobacillus; Probiotic; Microflora; VFA; Performance 